\title{
Abandoning the relativity of time
}

\section{Wagh S.M.}

Central India Research Institute, 34, Farmland, Ramdaspeth, Nagpur, India;

E-mail: Wagh <waghsm.ngp@gmail.com>;

Firstly, we discuss an error in the derivation of the "Standard" Doppler shift formula:

$v_{o}=v_{e} \sqrt{1-\beta^{2}} /(1+\beta \cos \theta)$. On correcting it, we then obtain $v_{o}=v_{e}(1-\beta \cos \theta) \sqrt{1-\beta^{2}}$. However, this "correct" special relativistic formula for the Doppler shift disagrees with the Ives-Stilwell experiment, and with all experiments supporting the standard formula. We therefore argue that the relativity of time is ruled out by experiments, and that the universality of time is upheld as the only possible alternative. Universal time also arises "naturally" within the categorical framework of the Universal Theory of Relativity.

Keywords: Correct Doppler formula, No relative time, Universal Time.

DOI: $10.18698 / 2309-7604-2015-1-537-545$

\section{Introduction}

While proposing special relativity, Einstein took [1-3] a bold and revolutionary step of proposing, also, the relativity of time.

Special relativity got eventual wide acceptance when its predictions agreed with results of the Ives-Stilwell experiment [4] and those of many cosmic ray experiments involving highly energetic charges emitting radiation [5].

Since then, the relativity of time; that time runs at different rates for observers differing in their states of motion; is the "implicit" basis of theories like quantum field theory [6], general relativity [7], unified field theory [8], string theory [9], quantum gravity [10], cosmology [11], etc.

Predictions of the relativistic quantum field theory (specifically, those of quantum electrodynamics and quantum chromo-dynamics) have been verified [6] in many particle accelerator experiments, importantly, to quite remarkable accuracies.

Notwithstanding aforementioned achievements of various theories involving the relativity of time, derivation of the standard Doppler shift formula using Lorentz transformations has an error that had gone unnoticed in the past.

Recently, we pointed out this error in Appendix of [12]. Therein, we had obtained "correct special relativistic formula" for the Doppler shift, also; and had shown that it disagrees with the Ives-Stilwell experiment [4]. For completeness here, we will recall the derivation of Doppler shift from [12]. 


\section{Deriving doppler shift}

Our further considerations are, then, based on Figure 1, showing in Part (a), a stationary source emitting "first" pulse at time $t_{1}$ and the "second" pulse at time $t_{2}$, time measured in the restframe of the source. The frequency of emission of the wave train is then $1 /\left(t_{2}-t_{1}\right)$. The "initial" line-of-sight, (SO), makes an angle $\theta$ with the $\mathrm{Y}$-axis; as is shown in Figure 1. When the first pulse reaches the observer at $\mathrm{O}$, time elapsed in the rest frame of the source is $T_{1}=t_{1}+(S O) / c$.
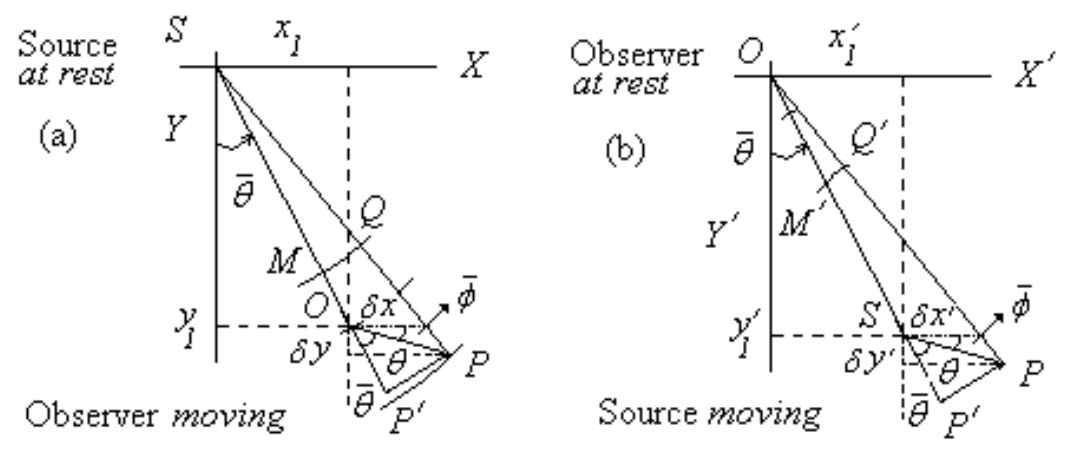

Fig. 1. Geometry for derivation of Doppler shift

Let the observer move along the line $O P$ making an angle $\bar{\phi}$ with the $X$-axis, with $P$ being the point at which the second pulse reaches the observer at time $T_{2}=t_{2}+(S P) / c$ in the rest-frame of the source. Then, we have

$$
T_{2}-T_{1}=t_{2}+(S P) / c-t_{1}-(S O) / c
$$

With $(S P)=\sqrt{\left(x_{1}+\delta x\right)^{2}+\left(y_{1}+\delta y\right)^{2}},(S O)=\sqrt{x_{1}{ }^{2}+y_{1}{ }^{2}}, x_{1}>>\delta x, y_{1}>>\delta y$, we get:

$$
T_{2}-T_{1} \approx t_{2}-t_{1}+\sin \bar{\theta} \delta x+\cos \bar{\theta} \delta y
$$

But, $\delta x=(O P) \cos \bar{\phi}$ and $\delta y=(O P) \cos \bar{\phi}$; and we get to first order of $\delta x$ and $\delta y$ :

$$
T_{2}-T_{1} \approx t_{2}-t_{1}+\frac{(O P)}{c} \sin (\bar{\theta}+\bar{\varphi}) \approx t_{2}-t_{1}+\frac{(O P)}{c} \cos \theta
$$




$$
c \Delta T \approx c \Delta t+(O P) \cos \theta
$$

where $\theta$ is the angle $O P$ makes with $S O$.

Now, consider the observer to be stationary and the source to be moving. Then, to first order of the infinitesimals $\delta x$ and $\delta y$, considerations similar to those of the above applied to Part (b) of Figure 1 lead to

$$
\begin{gathered}
T_{2}^{\prime}-T_{1}^{\prime} \approx t_{2}^{\prime}-t_{1}^{\prime}+\frac{(S P)}{c} \sin (\bar{\theta}+\bar{\varphi}) \approx t^{\prime}{ }_{2}-t^{\prime}{ }_{1}+\frac{(S P)}{c} \cos \theta \\
c \Delta T^{\prime} \approx c \Delta t^{\prime}+(O P) \cos \theta
\end{gathered}
$$

Time measured by the observer is distinguished here an overhead prime.

Importantly, we are not required to specify how the observer moves along line OP or the source moves along line SP, ie, with uniform velocity or uniform acceleration or variable acceleration. Equations (3) and (4) hold for any relative motion of the source and observer, generally; we note.

Now, for the case of uniform velocity that we denote as $u$, we may approximate (see Page 142 of [13]):

$$
(S P)=u \Delta t^{\prime}
$$

Using Eq. (5) in Eq. (4), we then obtain

$$
\Delta T^{\prime}=(1+\beta \cos \theta) \Delta t^{\prime} \Rightarrow 1 / \Delta T^{\prime} \equiv v_{o}=v_{e} \frac{\sqrt{1-\beta^{2}}}{1+\beta \cos \theta}
$$


where we have used $\Delta t^{\prime}=1 / v_{e} \sqrt{1-\beta^{2}}$, and that $\beta=u / c$, with $v_{e}$ being the frequency of emitted wave in the rest frame of the source. Eq. (6) is the standard formula for Doppler shift (or Eq. (517) on Page 143 of [13]).

On using the inverse Lorentz transformation in Eq. (3), we also obtain Eq. (6). This is therefore the manner in which the special theory of relativity ensures that the observer does not detect self-motion using Doppler Effect, then.

But, Eq. (5) is in error. Distance (SP) is "not" $u \Delta t$ ', but $(S P)=u \Delta T$ '. The Source (or the observer), if at all it does so, moves only during the period $\Delta T^{\prime}=T_{2}^{\prime}-T_{1}^{\prime}($ or $\Delta T$ ); and not during the period $\Delta t^{\prime}=t_{2}^{\prime}-t_{1}^{\prime}($ or $\Delta t)$.

We emphasize here that the time interval $\Delta t$ is that of the emission of successive pulses by the source, while the time interval $\Delta T$ is that of the reception of those pulses by the observer, moving or not.

Then, using $(S P)=u \Delta T^{\prime}$, the only correct expression, we obtain:

$$
\Delta T^{\prime}=\frac{\Delta t^{\prime}}{(1-\beta \cos \theta)} \Rightarrow 1 / \Delta T^{\prime} \equiv v_{o}=v_{e}(1-\beta \cos \theta) \sqrt{1-\beta^{2}}
$$

The same equation, Eq. (7), is also obtained using the inverse Lorentz transformation. Consequently, it is therefore ensured that the observer does not detect self-motion using Doppler Effect.

In terms of wavelengths, we obtain from Eq. (7):

$$
\lambda_{o}(\theta)=\frac{\lambda_{e}}{(1-\beta \cos \theta) \sqrt{1-\beta^{2}}}
$$

On calculating for $\theta=0$ and $\theta=\pi$, we obtain for the first and second order wavelength shifts from the above as:

$$
\Delta^{(1)} \lambda=\lambda_{e} \beta, \Delta^{(2)} \lambda=\frac{3}{2} \lambda_{e} \beta^{2} \Rightarrow \Delta^{(2)} \lambda=\frac{3}{2 \lambda_{e}}\left[\Delta^{(1)} \lambda\right]^{2}
$$


Clearly, Eq. (9) provides the parabolic relation of the first order and second order wavelength shifts, but with "three times" the coefficient as is implied by the standard formula, Eq. (6), for the Doppler shift.

Recall that Ives and Stilwell experiment [4] shows that:

$$
\Delta^{(2)} \lambda=\frac{1}{2 \lambda_{e}}\left[\Delta^{(1)} \lambda\right]^{2}
$$

Eq. (9) is therefore ruled out by the Ives-Stilwell experiment. So, theoretically "correct" result of special relativity as contained in Eq. (7) is inconsistent with the results of the Ives-Stilwell experiment. Also, all experiments; and there are many; supporting the "standard formula", Eq. (6), of the Doppler shift rule out Eq. (7), then.

Special relativity gets experimentally rejected, then. Certain "ugly" experimental facts have "killed" a beautiful idea of relative time, here.

\section{Implications}

With "universal" time; or time running at the same rate for all observers; being the "only" permissible alternative we set $\Delta T^{\prime}=\Delta T$ and $\Delta t^{\prime}=\Delta t$, now. Then, Eq. (3) and Eq. (4) yield the same Doppler shift whenever $(S P)=(O P)$. This is then the manner in which an observer does not detect self-motion using Doppler Effect, now.

For the situation of a uniform velocity u, we then obtain using either Eq. (3) or Eq. (4):

$$
v_{o}=v_{e}(1-\beta \cos \theta)
$$

an expression for the Doppler shift that, as can be very easily verified, disagrees with the result of the Ives-Stilwell experiment.

It may seem that the "universal time" is ruled out also by the Ives-Stilwell experiment and other cosmic ray related experiments, then.

But, the involved issue is not just of the velocity dependence of the Doppler shift; but of its acceleration dependence, also.

Recall that the distance traveled by the source (observer) as in Eq. (4) (Eq. (3)) involves its acceleration. We emphasize that the acceleration dependence of the Doppler shift has not been 
investigated, adequately, in interpreting experimental or observational results as in, eg, [4], [14], etc.

Then, we may think of the velocity dependence of the acceleration of a body for the possible resolution of this issue. That is to say, we may then assume velocity dependence of acceleration that leads to a formula for the Doppler shift agreeing with all the known experimental results.

To this end, recognizing also that it becomes difficult and difficult to accelerate a body at higher and higher velocity, we may postulate the velocity dependence of acceleration $a$ as:

$$
a \frac{d a}{d \beta}=-c a_{o} f(\beta)
$$

where $a_{o}$ is a constant, $\beta=u / c$ and $f(\beta)$ is some function. Then, we have:

$$
\frac{d \beta}{d t} \equiv \frac{a}{c}=\sqrt{-\frac{2 a_{o}}{c} \int_{0}^{\beta} f\left(\beta^{\prime}\right) d \beta^{\prime}+\frac{2 K_{1}}{c^{2}}} \equiv \sqrt{-A g(\beta)+B}
$$

where $K_{l}$ is a constant of integration, and we have also set here that $g(\beta)=\int_{0}^{\beta} f\left(\beta^{\prime}\right) d \beta^{\prime}$, $A=2 a_{o} / c$, and $B=2 K_{1} / c^{2}$.

In recognition of fundamental fact that a body of zero inertia moving with $\beta=1$ cannot be accelerated, we set $a \rightarrow 0$ for $\beta \rightarrow 1$.

Then, if we let $g(\beta) \rightarrow \alpha$ for $\beta \rightarrow 1$, we have $B=\alpha A=2 \alpha a_{o} / c$. Thus,

$$
\frac{d \beta}{d t}=\sqrt{\frac{2 a_{o}}{c}[\alpha-g(\beta)]}
$$

Therefore, we obtain:

$$
t-t_{o}=\int_{0}^{\beta} \frac{d \bar{\beta}}{\sqrt{A[\alpha-g(\bar{\beta})]}}
$$


with $t_{o}$ as a constant of integration. Here, time $t$ is that of the motion of the source or the observer, and was $T^{\prime}$ in Eq. (4) or $T$ in Eq. (3).

To proceed any further, we now need an explicit form of function $g(\beta)$ or of function $f\left(\beta^{\prime}\right)$. Using it, we perform the integration in Eq. (15) to obtain the equation as $t-t_{o}=G(\beta)$, where $G(\beta)$ denotes the result of the involved integration.

Thus, we obtain an equation in $\beta$, which can be solved to obtain the distance $(S P)$ traveled by the source or distance $(O P)$ traveled by the observer. It can then be used in Eq. (4) or Eq. (3) to obtain the Doppler shift formula that can be made consistent with experiments for a proper choice of function $f\left(\beta^{\prime}\right)$ or $g(\bar{\beta})$.

However, important is not "this" formula alone, but also are "reason(s)" for velocity dependence of acceleration. That is, any formula for velocity dependence of acceleration needs to be put on some sound physical basis as a resolution of the involved issue. Thence, some physical principles, than are meeting the eye and of genuine significance, appear to be involved here.

\section{Discussion}

In conclusion, Eq. (6), the standard formula for Doppler shift, is experimentally supported, but is theoretically inappropriate. Furthermore, correct special relativistic formula of Eq. (7) gets ruled out by the Ives-Stilwell experiment.

Now, notice that Eq. (7) has been obtained on transforming time as per Lorentz transformation. It is irrelevant whether we use Eq. (3) or Eq. (4) to obtain the Doppler shift, because it is also ensured that the observer does not detect, in any manner, self-motion using Doppler Effect.

Therefore, the relativity of time as proposed in special relativity is inconsistent with the Ives and Stilwell result, the very first instance indeed of a special relativistic formula contradicting any of the laboratory experiments. So, special relativity gets experimentally rejected here.

It is noteworthy that within the "most general mathematical framework" of category theory [15] as has been used to describe the physical concepts of universal relativity, universal time also arises in a natural manner [16]. Therefore, we had then also discussed [17-21] in some details, various implications of time being universal.

Then, with universal time and with the (temporally variable) acceleration of the source being included in the Doppler shift, the "energy budget" of the prime mover in AGNs and quasars is substantially non-relativistic, and the non-relativistic jets are of wide angular spread. Specifically, we find $[20,21]$ that the blue-shifted jet in micro-quasar SS433 has speed 0.022c and 
an angular spread of $\left[33^{\circ}, 133^{\circ}\right]$ with average angle of inclination of the jet to the line of sight being $86^{\circ}$. The red-shifted jet has speed of $0.29 \mathrm{c}$, angular spread of $\left[245^{\circ}, 255^{\circ}\right]$ (obtained from only six spectral lines) and an average angle of inclination of $248^{\circ}$ to the line of sight. These inferences are widely "different" than those obtained [22] using the standard formula for the Doppler shift. (Refer to talk by Dr. Sarwe in this conference.)

In Section §III, we discussed a plausible way to reconcile the theory of Doppler effect with results of experiments, with universal time that runs at the "same rate" for all observers. We imagined the velocity dependence of acceleration as being this reason. We also emphasized the need for physical justification of this assumption.

We avoid stating an explicit Doppler shift formula here without an appropriate and adequate discussion of reason(s) for the velocity dependence of acceleration. This will be the subject of an independent study providing for relevant details.

\section{References}

1. Einstein A. (1952). The Principle of Relativity: A collection of original papers on the special and general theory of relativity. New York: Dover.

2. Einstein A. (1968). Relativity: The Special and the General Theory. London: Methuen \& Co. Ltd.

3. Einstein A. (1970). Albert Einstein: Philosopher Scientist. La Salle: Open Court Publishing Company.

4. Ives H.E., Stilwell G.R. (1938). An experimental study of the Rate of a Moving Atomic Clock. J. Opt. Soc. Am., 28, 215-236.

5. Rossi B. (1939). The disintegration of mesotrons. Rev. Mod. Phys., 11, 296-303.

6. Itzykson C., Zuber J.B. (2005). Quantum Field Theory. New York: Dover.

7. Misner C., Thorne K.S., Wheeler J.A. (1973). Gravitation. San Francisco: Freeman.

8. Pais A. (1982). Subtle is the Lord ... The science and the life of Albert Einstein. Oxford: Clarendon Press.

9. Greene M.B., Schwarz J.H., Witten E. (1987). Superstring Theory. Cambridge: Cambridge University Press.

10. Narlikar, J.V., Dadhich N. (1997). Gravitation and Relativity: At the turn of the millennium. Proceedings of the GR-15 Conference.

11. Weinberg S. (1972). Gravitation and Cosmology. New York: John Wiley. 
12. Wagh S.M. (2014). Measuring velocity and acceleration using Doppler shift of a source with an example of Jet in SS433. J. of Astrophys \& Astron.

13. French A.P. (1966). Special Relativity. New York: W W Norton \& Co.

14. Ashby N. (2003). Relativity in the Global Positioning System. Living Rev. Relativity, 6, 1.

15. Maclane S. (1971). Categories for the Working Mathematicians. New York: Springer-Verlag.

16. Wagh S.M. (2011). Universal Relativity \& Categorical Dynamics. Tercentennary Conference of the Laplace-Runge-Lenz Vector.

17. Wagh S.M. (2013). On acceleration dependence of Doppler effect in light. Pramana, 81(3), 439-448.

18. Wagh S.M. (2013) Doppler effect, Gravity and Cosmology. J. Modern Physics, 4(8A), 102104.

19. Wagh S.M. (2013) Subtlety in relativity. Proceedings of SPIE - The International Society for Optical Engineering, 10, 8832.

20. Wagh S.M., Sarwe S.B. (2014). Jets in Micro-Quasar SS 433: Analysis involving Acceleration. J. Astrophys Aerospace Technol., 2:2.

21. Wagh S.M. (2014). Questioning the Relativity of Time. International Conference on Matters of Gravity and the Universe.

22. Marshall H.L., Canizares C.R., Hillwig T., Mioduszewski A., Rupen M., Schulz N.S., Nowak M., Heinz S. (2013). Multi-wavelength Observations of the SS433 Jets. arXiv, astroph.HE/1307.8427. 